\title{
Importance of circulating adipocytokines in multiple myeloma: a systematic review and meta-analysis based on case-control studies
}

\author{
Rui Liu', Dandan Gao' ${ }^{1}$ Yang Lv ${ }^{1}$, Meng Zhai ${ }^{1}$ and Aili He $e^{1,2^{*}}$
}

\begin{abstract}
Background: Adipocytes and their products, adipocytokines, play important roles in the generation and development of multiple myeloma (MM). Studies have demonstrated some adipocytokines to be associated with MM, although those results are controversial. Therefore, we conducted a meta-analysis to verify the association of adipocytokines with MM.

Methods: We performed a systematic retrieval of literature published prior to 26 October 2021. Standardized mean difference (SMD) with a 95\% confidence interval (CI) was calculated to evaluate pooled effects. Subgroup analysis and meta-regression analysis were conducted to detect sources of heterogeneity. Sensitivity analysis was performed to evaluate the stability of the study. Publication bias was assessed by funnel plots and Egger's linear regression test.

Results: Ten eligible studies with $1269 \mathrm{MM}$ patients and 2158 controls were included. The pooled analyses indicated that circulating leptin levels of MM patients were significantly higher than control levels (SMD $=0.87,95 \% \mathrm{Cl}: 0.33$ to 1.41), while the circulating adiponectin levels in MM patients were significantly lower than controls with a pooled SMD of -0.49 ( $95 \% \mathrm{Cl}:-0.78$ to -0.20$)$. The difference of circulating resistin levels were not significant between MM patients and controls (SMD $=-0.08,95 \% \mathrm{Cl}:-0.55$ to 0.39 ). Subgroup analysis and meta-regression analysis found that sample size, age, and sex were possible sources of heterogeneity. Sensitivity analysis demonstrated our pooled results to be stable.

Conclusion: Decreased circulating adiponectin and increased leptin levels were associated with the occurrence and development of MM. Adiponectin and leptin may be potential biomarkers and therapeutic targets for MM.
\end{abstract}

Keywords: Leptin, Adiponectin, Resistin, Multiple myeloma, Meta-analysis

\section{Introduction}

Multiple myeloma is a neoplastic plasma cell disease characterized by clonal proliferation of malignant mature $\mathrm{B}$ cells in bone marrow. Rapid proliferation of myeloma

\footnotetext{
${ }^{*}$ Correspondence: heaili@xjtu.edu.cn

${ }^{2}$ National-Local Joint Engineering Research Center of Biodiagnostics \& Biotherapy, The Second Affiliated Hospital of Xi'an Jiaotong University, Xi'an 710004, Shaanxi, China

Full list of author information is available at the end of the article
}

cells results in the accumulation of monoclonal proteins in the blood and urine. MM is the second most frequent hematological malignancy, accounting for $1.79 \%$ of all new cancer cases and $2.11 \%$ of all cancer deaths worldwide [1]. Older age, sex, African race, positive cancer history, and monoclonal gammopathy of undetermined significance (MGUS) are the recognized risk factors for MM [2]. A lifestyle-related factor considered to be an established risk factor for MM is obesity [3]. 
The bone marrow microenvironment plays an important role in the generation and progression of myeloma. The major component of BMME is the bone marrow adipose tissue (BMAT), which comprises $50-70 \%$ of BM volume and accounts for $5-10 \%$ of total fat [4]. It is well established that adipose tissue is not only an energy depot, but also an endocrine organ for the secretion of a number of adipocytokines: interleukin-6, tumor necrosis factor-alpha (TNF- $\alpha$ ), adiponectin, leptin, resistin, and visfatin, which regulate energy metabolism, hematopoiesis, inflammation and tumorigenesis [5]. Of note, adipocytes corrupted by myeloma cells not only boost tumor growth but also safeguard cancer cells from chemotherapy-induced apoptosis. Further, adipocytokines derived from "reprogrammed" adipocytes can skew the balance between osteoblasts and osteoclasts resulting in the development of myeloma-associated bone disease [6-8].

Leptin is a $16-k D a$ peptide hormone, found at increased levels in overweight individuals, regulates energy balance and suppresses appetite via the hypothalamus. Leptin is a pro-inflammatory and pro-angiogenic factor that regulates cell proliferation and immune responsiveness via paracrine signaling [9]. Conversely, adiponectin is negatively associated with body mass index (BMI). Adiponectin is a pleiotropic cytokine with the capacity to sensitize cell to insulin, produce anti-inflammatory effects and exert anti-neoplastic activities [10]. Resistin, as its name implies, can lead to resistance to the action of insulin, impairment of energy homeostasis and the development of diabetes mellitus. Moreover, resistin is an inflammatory regulator that induces the overexpression of TNF$\alpha$, IL-6, IL-12, and monocyte chemotactic protein-1 (MCP-1) by activating the NF- $\mathrm{KB}$ signaling pathway [11]. Visfatin, an obesity-related adipocytokine, has roles in angiogenesis, anti-apoptosis, and inducing inflammation. Its intracellular form is nicotinamide phosphoribosyl transferase (NAMPT), which is involved in the synthesis of nicotinamide adenine dinucleotide (NAD) [12]. Visfatin can function as a pre-B cell colony enhancing factor (PBEF) for B cell development [13].

Yoon et al. reported that circulating adiponectin and leptin were significantly associated with risks of obesityrelated cancers, such as endometrial cancer and breast cancer [14]. Furthermore, previous meta-analysis found that resistin and visfatin were significantly related to cancer risk $[15,16]$. Although several epidemiological experiments examined the association among these adipocytokines and $\mathrm{MM}$, results have been controversial and inconsistent, and are likely due to methodological and study population differences. Herein, we conducted a systematic review and meta-analysis that evaluated the role of adipocytokines in MM. The adipocytokines evaluated were leptin, adiponectin, resistin and visfatin.

\section{Materials and methods}

We followed the Preferred Reporting Items for Systematic Reviews and Meta-Analyses (PRISMA) 2009 checklist [17]. This study has been registered on PROSPERO with the registration number CRD42021228394.

\section{Search strategy}

Two investigators independently conducted a systematic literature retrieval in PubMed, Web of Science, and EMBASE to identify relevant studies prior to 26 October 2021. The search terms were as follows; "multiple myeloma" or "plasma cell neoplasm" combined with "adipokines" OR "adiponectin" OR "leptin" OR "resistin" OR "visfatin". In addition, we scrutinized the references of relevant articles and reviews manually to add additional studies to the meta-analysis. The detailed search strategy is shown in Supplementary Methods.

\section{Inclusion and exclusion criteria}

Publications were eligible if they met all of the following inclusion criteria: (1) case-control or cohort studies published as original articles, as well as other original articles containing relevant raw data; (2) studies evaluating associations among circulating adipocytokine levels and myeloma; (3) all patients were definitely diagnosed multiple myeloma; (4) sufficient data were available for the estimation of SMD with $95 \%$ CI.

The exclusion criteria were the following: (1) review papers, opinions, editorials, nonoriginal studies, interim analysis, pooled analysis, and studies published in multiple journals that were based on the same data; (2) conference abstracts that lacked baseline information and could not conduct methodological evaluations; (3) lack of the control group.

Two authors screened the relevant publications based on the above criteria. Discrepancies were resolved by discussion or consultation with another investigator.

\section{Data extraction}

We performed data extraction based on Meta-analysis of Observational Studies in Epidemiology (MOOSE) checklist [18]. Variables were extracted as follows: last name of the first author, publication year, study design, country, sample size, control source, age, sex, BMI, diagnostic criteria, recruiting time of samples, test method, types of adipocytokines, circulating adipocytokines (mean, standard deviation), and unit. Information for eligible publications was collected independently by two reviewers, with re-evaluation by the third reviewer, if there was disagreement. 


\section{Quality assessment}

The quality of the selected studies was evaluated by two independent reviewers based on the Newcastle-Ottawa Scale (NOS) [19]. Disagreements were resolved by discussion. Total scores ranged from 0 to 9 based on three perspectives: selection, comparability and exposure. A study with a score greater than 6 was identified as a highquality study. We used Grading of Recommendations, Assessment, Development, and Evaluations (GRADE) to assess the credibility of the current evidence [20].

\section{Statistical analysis}

SMD with $95 \%$ CI was calculated based on the sample size, mean, and standard deviation of all eligible studies to assess the association between circulating concentration of adipocytokines and multiple myeloma. However, a few studies only reported median, quartiles, or maximum, and minimum. For those studies, we contacted the corresponding author for detailed information or estimated the sample mean and standard deviation from the available data by statistical methods [21,22].

Heterogeneity was assessed by the chi-squared-based $Q$ test and $I^{2}$ statistics. Cut-offs of $P<0.10$ and $I^{2}>50 \%$ were considered to be statistically significant heterogeneity. The pooled SMD was calculated by the fixed-effect model (FEM) if the heterogeneity was not significant. Otherwise, a random-effect model (REM) was calculated. Subgroup analysis and meta-regression analysis were conducted to detect underlying sources of heterogeneity. Sensitivity analysis was performed to confirm the effects reported by individual studies and to evaluate the stability of meta-analysis results.

Publication bias was assessed qualitatively by use of funnel plots and quantitatively by Egger's linear regression test. Visual detection of asymmetry in funnel plots and a $P<0.05$ indicated statistically significant publication bias. With publication bias, Trim and Fill analysis was used to assess the number of missing studies. The pooled results were re-evaluated with the addition of missing studies [23].

All data analysis was conducted with StataMP 14 software and Review Manager 5.4 software.

\section{Results}

\section{Literature selection}

As shown in Fig. 1, the initial comprehensive search yielded 392 articles based on the search strategy (58 articles from PubMed, 143 articles from the Web of Science, 191 articles from EMBASE), of which 106 articles were excluded due to duplication. After scanning titles and abstracts, 255 articles were ruled out because of apparent irrelevance. More detailed selection resulted in 21 papers. Finally, 10 articles met the inclusion-exclusion criteria and were eligible for the meta-analysis [24-33].

\section{Study characteristics}

The primary characteristics of all included studies are summarized in Table 1. A total of 1269 MM cases and 2158 age-matched controls were evaluated. Ten studies were conducted in six countries (two in Greece, three in USA, two in China, one in Turkey, one in Egypt, and one in Iraq). The age of most participants was between 60 and 65 years. No significant difference in age was observed between MM patients and controls. Nine of ten studies considered the impact of BMI on adipocytokine levels, and all individuals had a mean BMI well below the standard of WHO for obesity (BMI $\geq 30.0 \mathrm{~kg} / \mathrm{m}^{2}$ ). All studies were case-control studies, with two nested case-control studies. A total of four different adipocytokines were evaluated. Seven studies included leptin [24-27, 30, 32], five studies included adiponectin [26, 27, 29, 30, 32], six studies included resistin [25, 26, 28, 30, 31, 33], and two studies included visfatin [30, 32]. Five of ten studies were scored above six and were considered as high-quality studies according to NOS. The detailed scoring process is shown in Table S1. According to GRADE assessment, all eligible studies were observative research without pluses and minuses, and were graded low. The detailed table is presented in Table S2.

\section{Association of adipocytokines with MM}

Detailed statistics for each adipocytokine are presented in Table 2 . The statistics of ten studies were analyzed by REM to compare different circulating levels of adipocytokine in MM patients and controls.

Five out of seven studies found MM patients to have significantly increased circulating leptin concentrations compared to controls. As shown in Fig. 2A, a total of $406 \mathrm{MM}$ patients and 530 controls were included in the pooled analysis. Pooled results showed a significantly higher level of leptin in patients with MM than in controls (SMD $=0.87,95 \mathrm{CI}$ : 0.33 to $1.41, z=3.14, P$ $=0.002)$. However, statistically significant heterogeneity was observed in these studies $\left(P<0.00001, I^{2}=90 \%\right.$ 95\%CI: 0.8250 to 0.9458 ).

For adiponectin levels, significant reductions in four of five studies were found in patients with MM. $938 \mathrm{MM}$ patients and 1715 controls were analyzed for adiponectin. Circulating adiponectin levels in MM patients were significantly lower than in controls with a pooled SMD of -0.49 (95\%CI: -0.78 to $-0.20, z=3.27, P=0.001$; Fig. 2B). Similarly, significant heterogeneity was observed among studies $\left(P<0.0001, I^{2}=84 \%, 95 \%\right.$ CI: 0.6507 to 0.9304$)$. 



Records after duplicates removed ( $n=286$ )

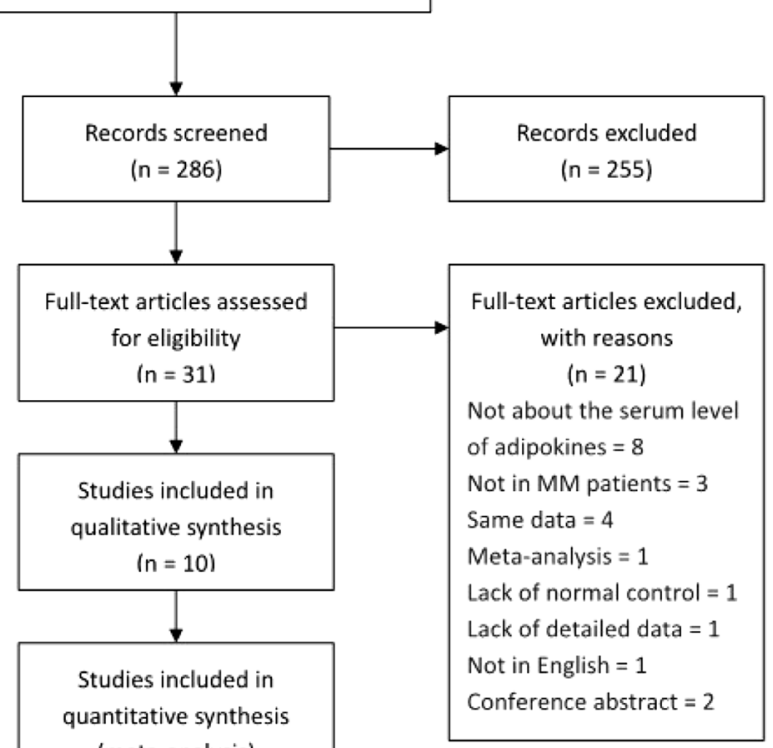

Fig. 1 Flow diagram of the selection process for the meta-analysis

Two of six studies observed that MM patients had significantly altered circulating resistin level compared with controls. Pooled analysis showed that circulating resistin levels were not significantly different for $367 \mathrm{MM}$ patients and 524 controls (SMD $=-0.08,95 \% \mathrm{CI}$ : -0.55 to $0.39, z=0.34, P=0.73)$. Significant heterogeneity was found among these five studies $\left(P<0.0001, I^{2}=87 \%\right.$, 95\%CI: 0.7394 to 0.9349; Fig. 2C).

Studies of visfatin were limited by sample size, with diametrically opposed conclusions drawn. Those studies were not included in the pooled analysis.

\section{Subgroup analysis and meta-regression}

Subgroup analysis based on sample size, age, and BMI was performed to identify potential sources of leptin heterogeneity (Fig. 3). First, higher levels of circulating leptin were observed in overweight $(\mathrm{SMD}=0.49,95 \% \mathrm{CI}$ : 0.06 to 0.93 , Fig. $3 \mathrm{~A}$ ) and normal weight $(\mathrm{SMD}=2.27,95 \% \mathrm{CI}$ : 0.68 to 3.86, Fig. 3A) MM patients than in controls. Second, compared with controls, MM patients under the age of 60 years had significantly higher levels of leptin (SMD
$=1.84,95 \%$ CI: 0.87 to 2.82 , Fig. $3 \mathrm{~B}$ ), while those over 60 did not (SMD $=0.27$, 95\%CI: -0.06 to 0.59 , Fig. 3B). Furthermore, in the subgroup of case size less than 50, MM patients had significantly higher leptin levels than controls $(\mathrm{SMD}=1.39,95 \% \mathrm{CI}$ : 0.27 to 2.50 , Fig. $3 \mathrm{C}$ ), which was not true in the subgroup of case size more than 50 (SMD $=0.34$, 95\%CI: -0.06 to 0.74 , Fig. 3C). Next, metaregression analysis was performed to assess the impact of sample size, age, sex, region, BMI, study design, and NOS quality on SMD. Sex $(P=0.018)$ was found to be a significant contributing factor for between-study variance, while sample size $(P=0.262)$, age $(P=0.072)$, region $(P$ $=0.070)$, BMI $(P=0.246)$, study design $(P=0.380)$, and NOS quality $(P=0.642)$ did not significantly affect study variance.

Subgroup analysis of adiponectin is summarized in Fig. 4. With regard to race, both Caucasian and Asian MM patients had significantly lower circulating adiponectin levels than controls (Fig. 4A). No matter the case size, decreased adiponectin levels were found in MM patients (SMD $=-0.17,95 \% \mathrm{CI}:-0.25$ to -0.08 ; SMD 


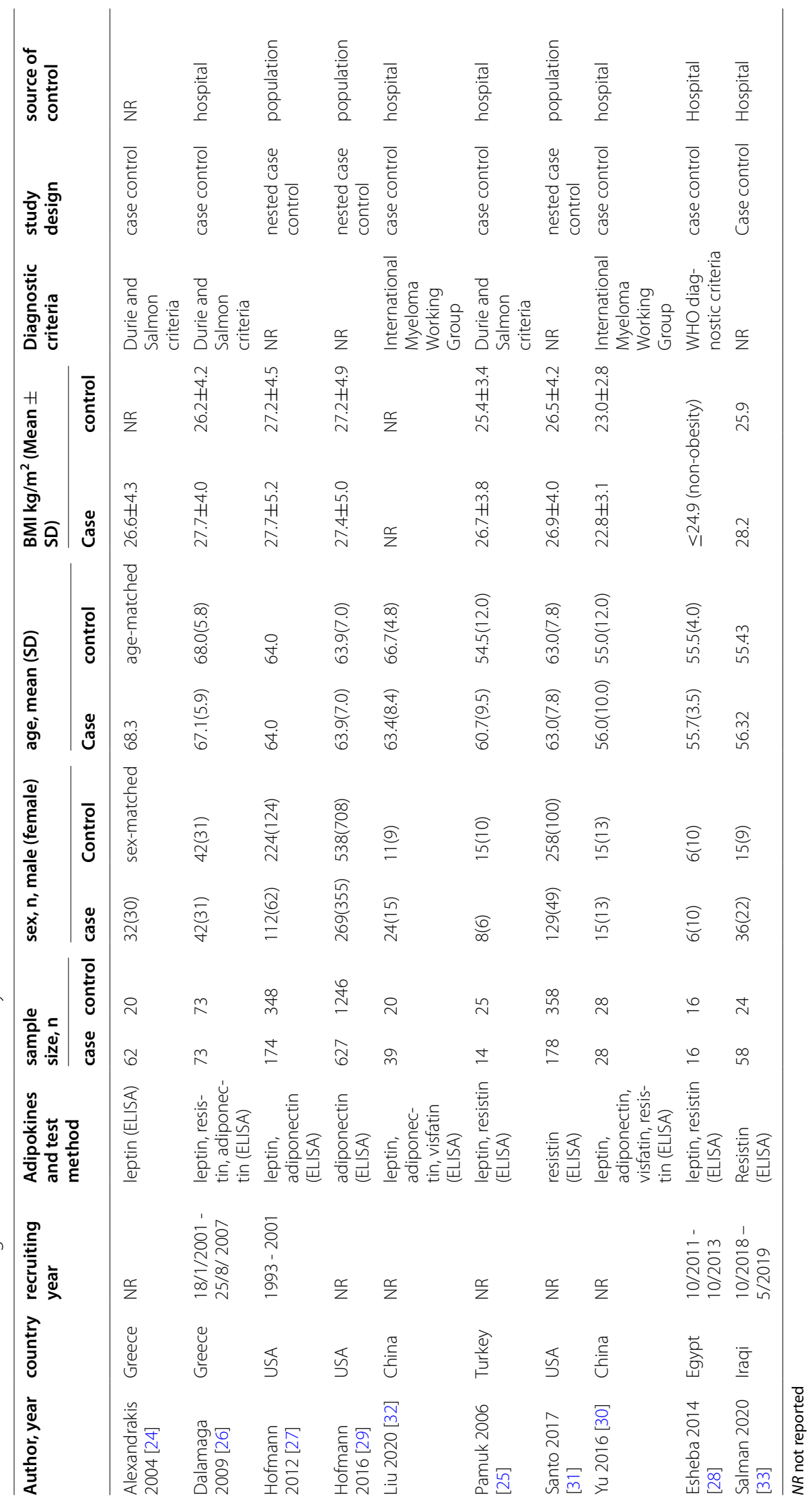


Table 2 Circulating levels of leptin, adiponectin, resistin and visfatin in MM patients and controls

\begin{tabular}{|c|c|c|c|c|c|c|c|c|}
\hline \multirow[t]{2}{*}{ Author, year } & \multicolumn{3}{|l|}{ Case } & \multicolumn{3}{|c|}{ Control } & \multirow[t]{2}{*}{ Unit } & \multirow[t]{2}{*}{$P$ value } \\
\hline & Mean & SD & $\mathbf{N}$ & Mean & SD & $\mathbf{N}$ & & \\
\hline \multicolumn{9}{|l|}{ Circulating leptin levels } \\
\hline Alexandrakis 2004 [24] & 17.96 & 20.39 & 62 & 4.18 & 0.96 & 20 & $\mathrm{ng} / \mathrm{ml}$ & $<0.0001$ \\
\hline Pamuk 2006 [25] & 22.6 & 14.7 & 14 & 10.3 & 7.6 & 25 & $\mathrm{ng} / \mathrm{ml}$ & $<0.01$ \\
\hline Dalamaga 2009 [26] & 27.5 & 17.6 & 73 & 21.9 & 9.5 & 73 & $\mathrm{ng} / \mathrm{ml}$ & 0.02 \\
\hline Hofmann 2012 [27] & 15.76 & 16.83 & 174 & 15.36 & 16.92 & 348 & $\mathrm{ng} / \mathrm{ml}$ & NS \\
\hline Esheba 2014 [28] & 5.82 & 1.43 & 16 & 2.16 & 0.72 & 16 & $\mathrm{ng} / \mathrm{ml}$ & $<0.0001$ \\
\hline Hu 2016 & 6.82 & 3.09 & 28 & 2.91 & 1.81 & 28 & $\mathrm{ng} / \mathrm{ml}$ & $<0.01$ \\
\hline Liu 2020 [32] & 0.21 & 0.11 & 39 & 0.21 & 0.13 & 20 & $\mathrm{ng} / \mathrm{ml}$ & NS \\
\hline \multicolumn{9}{|c|}{ Circulating adiponectin levels } \\
\hline Dalamaga 2009 [26] & 14.3 & 7.3 & 73 & 21.7 & 10.3 & 73 & $\mathrm{ng} / \mathrm{ml}$ & $<0.0001$ \\
\hline Hofmann 2012 [27] & 10.42 & 6.98 & 174 & 11.68 & 7.84 & 348 & $\mu \mathrm{g} / \mathrm{ml}$ & NS \\
\hline Hofmann 2016 [29] & 12 & 6.3 & 624 & 13.1 & 6.8 & 1246 & $\mu \mathrm{g} / \mathrm{ml}$ & $<0.05$ \\
\hline Hu 2016 & 5.79 & 2.37 & 28 & 9.29 & 3.45 & 28 & $\mu \mathrm{g} / \mathrm{ml}$ & $<0.01$ \\
\hline Liu 2020 [32] & 12.37 & 3.13 & 39 & 13.8 & 0.95 & 20 & $\mathrm{ng} / \mathrm{ml}$ & $<0.05$ \\
\hline \multicolumn{9}{|l|}{ Circulating resistin levels } \\
\hline Pamuk 2006 [25] & 3.3 & 3.3 & 14 & 1.97 & 0.6 & 25 & $\mathrm{ng} / \mathrm{ml}$ & NS \\
\hline Dalamaga 2009 [26] & 9.4 & 5 & 73 & 15.9 & 6.8 & 73 & $\mathrm{ng} / \mathrm{ml}$ & $<0.0001$ \\
\hline Esheba 2014 [28] & 1.56 & 0.74 & 16 & 1.6 & 0.68 & 16 & $\mathrm{ng} / \mathrm{ml}$ & 0.438 \\
\hline Hu 2016 & 8.98 & 6.41 & 28 & 9.48 & 6.18 & 28 & $\mathrm{ng} / \mathrm{ml}$ & 0.091 \\
\hline Santo 2017 [31] & 5.56 & 2.26 & 178 & 5.89 & 2.16 & 358 & $\mathrm{ng} / \mathrm{ml}$ & NS \\
\hline Salma 2020 [33] & 1.967 & 3.595 & 58 & 0.604 & 0.622 & 24 & $\mathrm{ng} / \mathrm{ml}$ & 0.009 \\
\hline \multicolumn{9}{|l|}{ Circulating visfatin levels } \\
\hline Yu 2016 [30] & 8.35 & 5.06 & 28 & 7.74 & 4.79 & 28 & $\mathrm{ng} / \mathrm{ml}$ & 0.819 \\
\hline Liu 2020 [32] & 102.76 & 90.41 & 39 & 22.55 & 21.41 & 20 & $\mathrm{ng} / \mathrm{ml}$ & $<0.05$ \\
\hline
\end{tabular}

NS not significant

$=-0.83,95 \% \mathrm{CI}=-1.12$ to -0.54 , Fig. $4 \mathrm{~B})$. Meta-regression analysis identified study design $(P=0.016)$ to be a significant contributing factor to heterogeneity, but sample size $(P=0.215)$, age $(P=0.440)$, region $(P=0.094)$, BMI $(P=0.197)$, sex $(P=0.919)$ and NOS quality $(P=$ $0.665)$ were not.

\section{Sensitivity analysis}

Studies were individually excluded to evaluate the robustness of the results and the influence of each study on pooled SMD (Fig. 5A and B; Tables S3 and S4). The omission of any particular study did not appreciably change combined SMD. Estimates in each case were well within the confidence limits of the overall estimates, which implied the stability of results.

\section{Publication bias}

For adiponectin there were only five eligible studies, and thus, Egger's regression test rather than funnel plot was used to assess publication bias. No evidence of publication bias was found $(P=0.061)$. For leptin, evident asymmetry was observed in the funnel plot (Fig. S1), with an Egger's test result $(P=0.01)$, indicating the significant publication bias. Therefore, Trim and Fill analysis was used to evaluate the effect of publication bias on pooled results. Analysis showed the imputed pooled results were identical to original results (SMD $=0.890,95 \%$ CI: 0.337 to 1.442). No missing studies were added to the filled funnel plot (Fig. S2).

\section{Discussion}

$\mathrm{MM}$ is an incurable neoplasm. Even though overall survival for MM patients has increased, patients' medical needs remain unmet. It is therefore important to explore underlying oncogenic mechanisms and novel predictive biomarkers that will allow the individualized cancer therapies for specific patients. Obesity has been well established as a risk factor for hypertension, cardiovascular disease, and type-2 diabetes mellitus. Accumulating epidemiological studies have demonstrated obesity to be a risk factor for cancers, including MM [34]. Obesityrelated oncogenic mechanisms are involved in two major aspects: insulin resistance and chronic inflammation $[35$, 




36], which are tightly associated with the secretion of adipocytokines.

Previous meta-analysis of a number of cancer patients demonstrated adiponectin and leptin to be associated with cancer generation. However, that analysis included only two MM studies [14, 37]. Moreover, the results of studies conducted to explore the associations among MM and adipocytokines have been inconsistent and limited. Therefore, we performed this meta-analysis to evaluate the associations of circulating levels of adiponectin, leptin, resistin, and visfatin with MM. The results suggested that increased circulating levels of leptin and decreased circulating levels of adiponectin were associated with a higher risk of MM. Circulating resistin levels did not differ between MM patients and controls. Due to the limited number of eligible studies, pooled analysis of visfatin was not conducted. Sensitivity analysis by sequential omission of individual studies produced statistically consistent results, which indicated the robustness of our meta-analysis. Moreover, adiponectin and leptin results are consistent with previous meta-analyses of renal cell carcinoma, breast, and colorectal cancer, which further supports the credibility of our results [14, 38-40]. In myeloma-associated bone disease, reprogrammed adipocytes increase leptin secretion while decrease adiponectin secretion to intensify osteoclastogenesis and to retard osteoblastogenesis, which is consistent with our pooled results [6]. These findings suggest that adipocytokine dysregulation affects bone marrow microenvironment and that the reprogrammed profile of adipocytokines may serve as novel promising indicator for myelomaassociated bone disease.

Circulating levels of adiponectin and leptin are in accordance with their cancer biological function. Leptin is an oncogenic factor due to its pro-inflammatory and pro-angiogenic effects. Yu et al. [41] demonstrated adipocyte-derived leptin to promote self-renewal of breast cancer stem cells and to induce chemo-resistance via the JAK/STAT3/CPT1B axis. Moreover, leptin is involved in the $\mathrm{PI} 3 \mathrm{~K} / \mathrm{AKT} / \mathrm{lysyl}$ hydroxylase signaling pathway, 


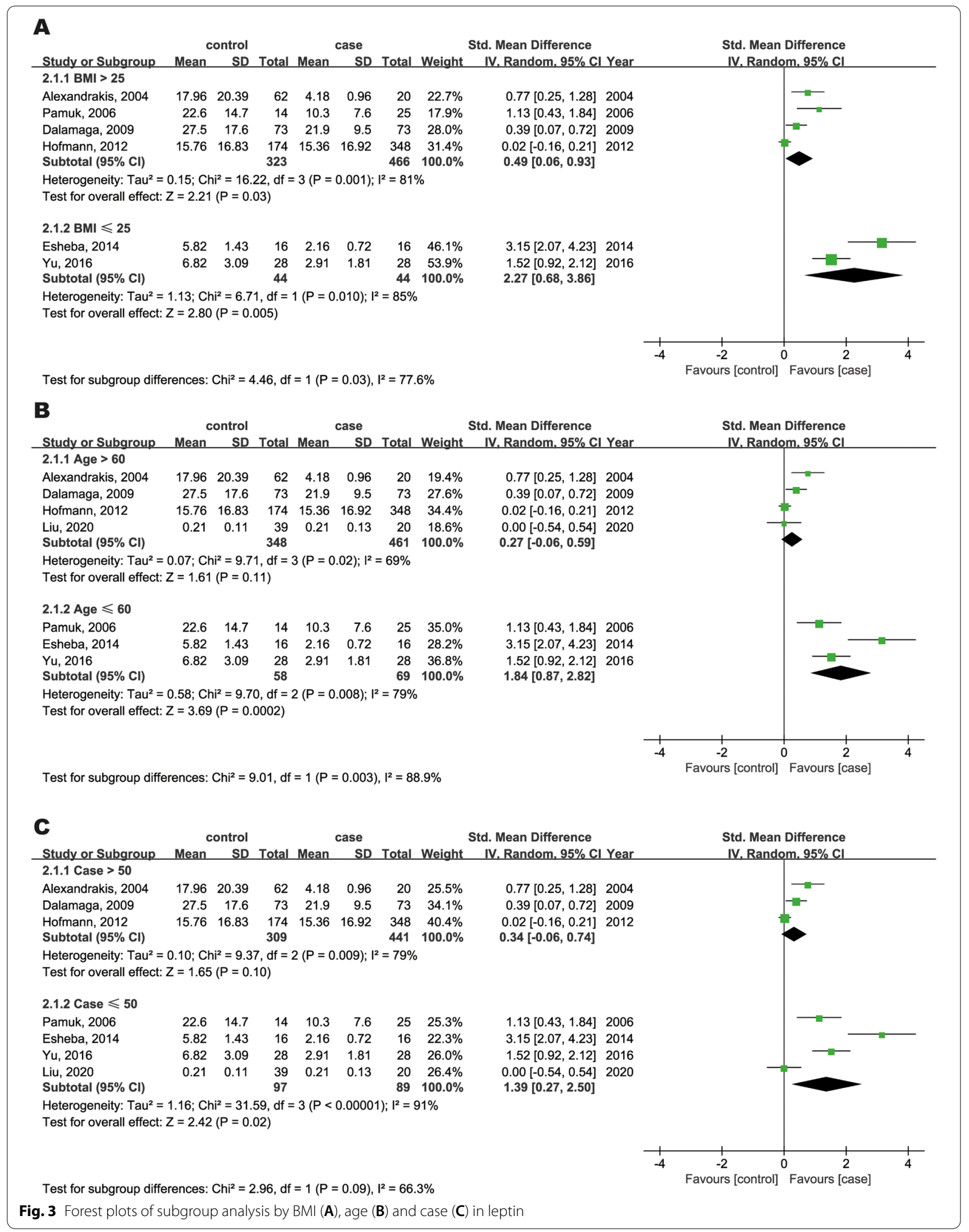




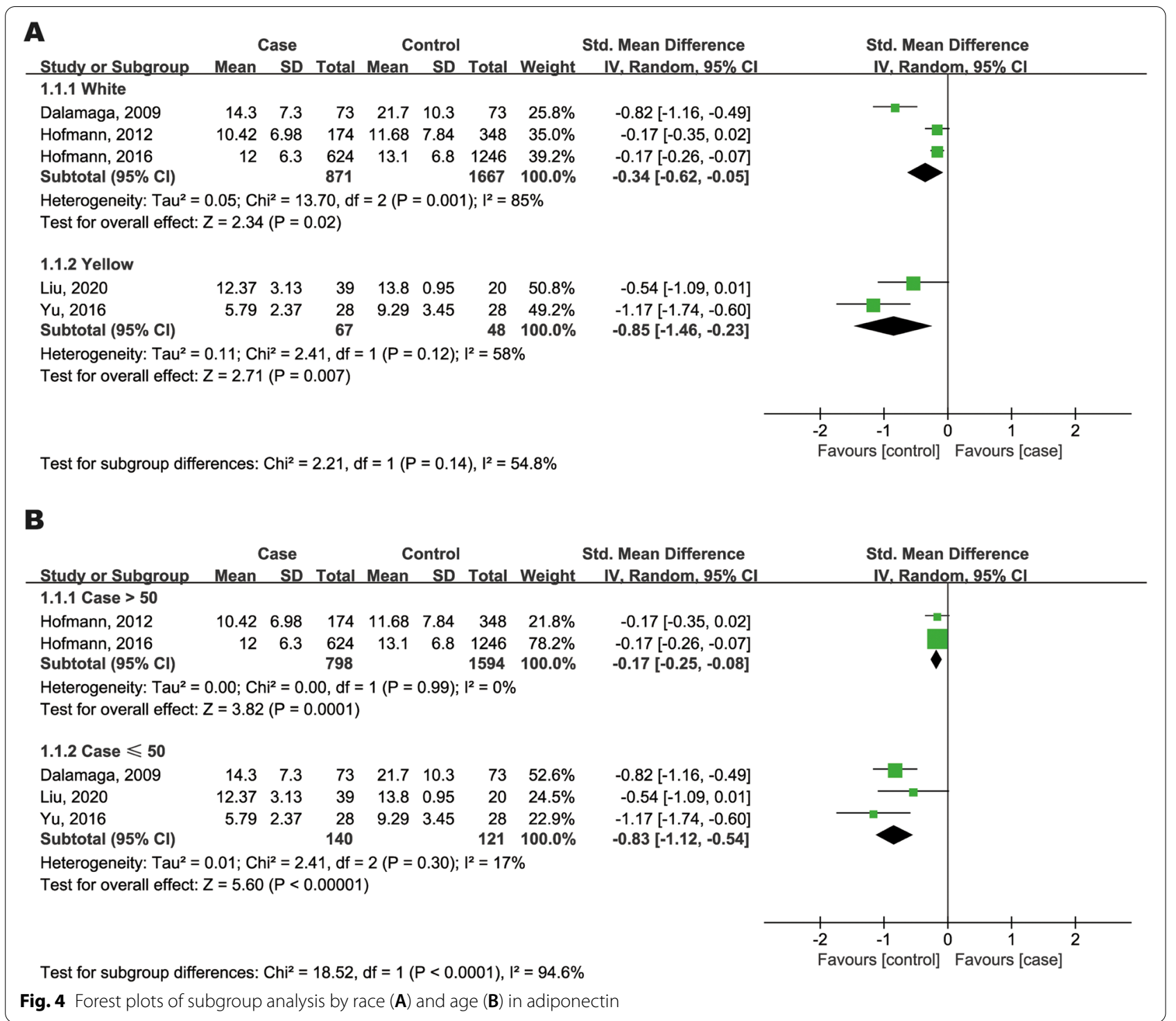

which facilitates breast cancer metastasis [42]. For MM, leptin plays a significant role in drug resistance by activating the JAK/STAT and PI3K pathways, enhancing proliferation and autophagy [30, 43].

Adiponectin has been identified as a "friendly" cytokine owing to its anti-tumor activity in breast cancer and colorectal cancer [44]. Several studies have demonstrated its tumor-suppressive activity in MM by inducing apoptosis [45]. Further, adiponectin can alleviate myeloma bone disease by inhibiting the differentiation and maturation of osteoclasts by activation of the mTOR pathway [32].

Resistin may play an oncogenic role in MM by serving as an inflammatory regulator. Previous pooled analysis demonstrated increased resistin circulating levels to be associated with a higher risk for colorectal cancer [46].
For breast cancer, resistin is involved in epithelial-mesenchymal transition and stemness, which are critical to metastasis and tumorigenesis via NF-kB/STAT3 pathways [47]. For MM, resistin can induce multidrug tolerance (melphalan, bortezomib, and carfilzomib) through NF-kB and PI3K/Akt pathways. Further, resistin can accelerate drug expulsion, epigenetically, via the DNA methyltransferases DNMT/ATP-binding cassette (ABC) transporter axis [48].

Visfatin is a significant adipocytokine due to its NAMPT activity, which is essential for the synthesis of NAD. Visfatin acts as an oncogenic factor by promoting proliferation, metastasis, angiogenesis, and drug resistance [49] in various solid tumors, including breast [50, 51], colorectal [52], and ovarian cancer [53]. Although 

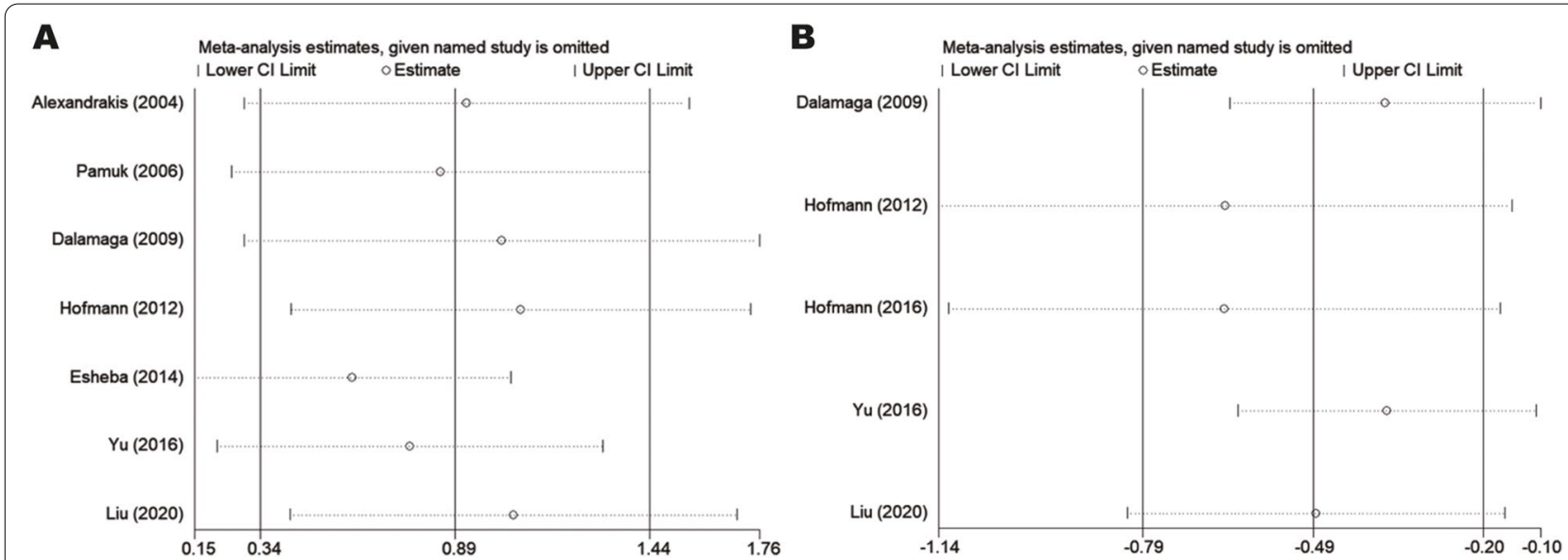

Fig. 5 The pooled SMD and $95 \% \mathrm{Cl}$ of eligible studies of leptin and adiponectin through sensitivity analysis

studies in MM are limited, visfatin has been reported to be involved in myeloma bone disease and myeloma cell growth. Further, the depletion of intracellular $\mathrm{NAD}^{+}$sensitizes bortezomib-induced cytotoxicity, indicating that visfatin could be a biomarker or therapeutic target for MM [54-56].

Heterogeneity was found in our pooled analyses. Subgroup analysis and meta-regression were used to identify the source of heterogeneity. Sample size, age, and sex were found to be possible sources of the heterogeneity for leptin. Study design might be the cause of heterogeneity for adiponectin. Furthermore, heterogeneity may be accounted for by the following clinical differences in MM studies. First, there may be differences in the demographic features and genetic background of Asian and Caucasian populations. Second, myeloma types, different stages, risk stratification, and tumor heterogeneity may be responsible. Third, non-myeloma related adipocytokine alterations such as BMI, past medical history, hormone receptor expression, menopausal status, and emerging endocrine diseases may have influenced the results. Another possible reason for heterogeneity may be that levels of adipocytokines vary by time of day, and a single blood sample measurement might be inadequate. Several methodological discrepancies were also observed. To avoid the effect of confounding factors, results of included research were adjusted for covariates such as sex and age, while few were adjusted for BMI. Furthermore, the source of controls was from hospital in eight of ten studies, but only three studies reported the strict exclusion criteria (such as obesity, diabetes, and hyperlipidemia). Thus, we suggest that future studies focused on adipocytokine should adjust for BMI, or make strict inclusion and exclusion criteria to avoid not cancerrelated impact on adipocytokine levels.
There are several limitations in this meta-analysis. First, all included studies were case-control, with inherent selection, information, and confounding bias. Second, data regarding myeloma types, cancer stage, risk stratification, past medical history, and lifestyle was not available. Third, the sample sizes of few eligible studies are small.

In conclusion, results herein suggest that low circulating levels of adiponectin and high circulating levels of leptin are associated with an increased risk for MM, indicating that adiponectin and leptin may be potential biomarkers.

\section{Abbreviations}

BMAT: Bone marrow adipose tissue; BMI: Body mass index; Cl: Confidence interval; ELISA: Enzyme-linked immunosorbent assay; FEM: Fixed-effect model; MGUS: Monoclonal gammopathy of undetermined significance; MM: Multiple myeloma; REM: Random-effect model; SMD: Standardized mean difference; TNF: Tumor necrosis factor.

\section{Supplementary Information}

The online version contains supplementary material available at https://doi. org/10.1186/s12902-022-00939-2.

Additional file 1: Table S1. Newcastle-Ottawa scale for the assessment of included studies. Table S2. Using GARDE to assess the credibility of the current evidence. Table S3. The pooled SMD and $95 \% \mathrm{Cl}$ of the eligible studies of leptin through sensitivity analysis. Table S4. The pooled SMD and $95 \% \mathrm{Cl}$ of the eligible studies of adiponectin through sensitivity analysis. Figure S1. Funnel plot for publication bias of the association between serum leptin levels and myeloma. Evident asymmetry was observed in the funnel plot, and the Egger's test result was $P=0.013$, which indicates the existence of significant publication bias. Figure S2. Filled Funnel plot of the association between serum leptin levels and myeloma by the use of Trim and Fill method. Trim and Fill analysis was used to evaluate the effect of publication bias on the pooled result. The analysis showed the imputed pooled result was identical to our original result $(\mathrm{SMD}=0.890,95 \% \mathrm{Cl}$ : 0.337 to 1.442 ). No missing studies were added to the filled funnel plot. 


\section{Acknowledgements}

We gratefully thank professor Jinsong Hu from Department of Cell Biology and Genetics, Xi'an Jiaotong University Health Science Center for valuable comments and critical revision of the manuscript. We gratefully thank professor Hofmann Jonathan from the Division of Cancer Epidemiology and Genetics, National Cancer Institute, Bethesda for valuable support of the detailed statistics. We thank International Science Editing (http://www.internationalscience editing.com) for editing this manuscript.

\section{Authors' contributions}

Conceptualization: ALH, RL. Methodology: RL, YL, DDG, MZ. Data curation: DDG, MZ, YLD. Formal analysis: RL, DDG. Software: RL. Original draft: RL. Supervision: ALH, DDG, MZ. Project administration: ALH, RL. The author(s) read and approved the final manuscript.

\section{Funding}

This research did not receive any specific grant from funding agencies in the public, commercial, or not-for-profit sectors.

\section{Availability of data and materials}

All data analyzed during this study are included in this published article.

\section{Declarations}

\section{Ethics approval and consent to participate}

Not applicable.

\section{Consent for publication}

Not applicable.

\section{Competing interests}

The authors declare that the research was conducted in the absence of any commercial or financial relationships that could be construed as a potential conflict of interest.

\section{Author details}

${ }^{1}$ Department of Hematology, The Second Affiliated Hospital of Xi'an Jiaotong University, 157, 5th West Road, Xi'an 710004, Shaanxi, China. ${ }^{2}$ National-Local Joint Engineering Research Center of Biodiagnostics \& Biotherapy, The Second Affiliated Hospital of Xi'an Jiaotong University, Xi'an 710004, Shaanxi, China.

Received: 21 September 2021 Accepted: 14 January 2022

Published online: 25 January 2022

\section{References}

1. Siegel RL, Miller KD, Goding Sauer A, Fedewa SA, Butterly LF, Anderson JC, et al. Colorectal cancer statistics, 2020. CA Cancer J Clin. 2020;70(3):145-64.

2. Dutta AK, Fink JL, Grady JP, Morgan GJ, Mullighan CG, To LB, et al. Subclonal evolution in disease progression from MGUS/SMM to multiple myeloma is characterised by clonal stability. Leukemia. 2019;33(2):457-68.

3. Abar L, Sobiecki JG, Cariolou M, Nanu N, Vieira AR, Stevens C, et al. Body size and obesity during adulthood, and risk of lympho-haematopoietic cancers: an update of the WCRF-AICR systematic review of published prospective studies. Ann Oncol. 2019;30(4):528-41.

4. Veldhuis-Vlug AG, Rosen CJ. Clinical implications of bone marrow adiposity. J Intern Med. 2018;283(2):121-39.

5. Masarwi M, DeSchiffart A, Ham J, Reagan MR. Multiple myeloma and fatty acid metabolism. JBMR Plus. 2019;3(3):e10173.

6. Liu H, He J, Koh SP, Zhong Y, Liu Z, Wang Z, et al. Reprogrammed marrow adipocytes contribute to myeloma-induced bone disease. Sci Transl Med. 2019;11(494):eaau9087.

7. Fairfield H, Dudakovic A, Khatib CM, Farrell M, Costa S, Falank C, et al. Myeloma-modified adipocytes exhibit metabolic dysfunction and a senescence-associated secretory phenotype. Cancer Res. 2021:81(3):634-47.

8. Fairfield H, Costa S, Falank C, Farrell M, Murphy CS, D'Amico A, et al. Multiple myeloma cells alter adipogenesis, increase senescence-related and inflammatory gene transcript expression, and alter metabolism in preadipocytes. Front Oncol. 2020;10:584683.

9. Favreau M, Menu E, Gaublomme D, Vanderkerken K, Faict S, Maes K, et al. Leptin receptor antagonism of iNKT cell function: a novel strategy to combat multiple myeloma. Leukemia. 2017;31(12):2678-85.

10. Fang $\mathrm{H}$, Judd RL. Adiponectin regulation and function. Comprehen Physiol. 2018;8(3):1031-63.

11. Sudan SK, Deshmukh SK, Poosarla T, Holliday NP, Dyess DL, Singh AP, et al. Resistin: An inflammatory cytokine with multi-faceted roles in cancer. Biochim Biophys Acta Rev Cncer. 2020;1874(2):188419.

12. Nacarelli T, Lau L, Fukumoto T, Zundell J, Fatkhutdinov N, Wu S, et al. $\mathrm{NAD}(+)$ metabolism governs the proinflammatory senescence-associated secretome. Nat Cell Biol. 2019;21(3):397-407.

13. Kumari B, Yadav UCS. Adipokine visfatin's role in pathogenesis of diabesity and related metabolic derangements. Curr Mol Med. 2018;18(2):116-25.

14. Yoon YS, Kwon AR, Lee YK, Oh SW. Circulating adipokines and risk of obesity related cancers: A systematic review and meta-analysis. Obes Res Clin Pract. 2019;13(4):329-39.

15. Mohammadi M, Mianabadi F, Mehrad-Majd H. Circulating visfatin levels and cancers risk: a systematic review and meta-analysis. J Cell Physiol. 2019;234(4):5011-22.

16. Gong WJ, Zheng W, Xiao L, Tan LM, Song J, Li XP, et al. Circulating resistin levels and obesity-related cancer risk: a meta-analysis. Oncotarget. 2016;7(36):57694-704

17. Moher D, Liberati A, Tetzlaff J, Altman DG. Preferred reporting items for systematic reviews and meta-analyses: the PRISMA statement. BMJ. 2009;339:b2535.

18. Stroup DF, Berlin JA, Morton SC, Olkin I, Williamson GD, Rennie D, et al. Meta-analysis of observational studies in epidemiology: a proposal for reporting. Meta-analysis Of Observational Studies in Epidemiology (MOOSE) group. Jama. 2000;283(15):2008-12.

19. Stang A. Critical evaluation of the Newcastle-Ottawa scale for the assessment of the quality of nonrandomized studies in meta-analyses. Eur J Epidemiol. 2010;25(9):603-5.

20. Atkins $D$, Best $D$, Briss PA, et al. Grading quality of evidence and strength of recommendations. BMJ. 2004;328(7454):1490. https://doi. org/10.1136/bmj.328.7454.1490.

21. Wan X, Wang W, Liu J, Tong T. Estimating the sample mean and standard deviation from the sample size, median, range and/or interquartile range. BMC Med Res Methodol. 2014;14:135.

22. Luo D, Wan X, Liu J, Tong T. Optimally estimating the sample mean from the sample size, median, mid-range, and/or mid-quartile range. Stat Methods Med Res. 2018;27(6):1785-805.

23. Peters $J$, Sutton AJ, Jones DR, Abrams KR, Rushton L. Performance of the trim and fill method in the presence of publication bias and between-study heterogeneity. Stat Med. 2007;26(25):4544-62.

24. Alexandrakis MG, Passam FH, Sfiridaki A, Pappa CA, Moschandrea JA, Kandidaki $E$, et al. Serum levels of leptin in multiple myeloma patients and its relation to angiogenic and inflammatory cytokines. Int J Biol Markers. 2004;19(1):52-7.

25. Pamuk GE, Demir M, Harmandar F, Yesil Y, Turgut B, Vural O. Leptin and resistin levels in serum of patients with hematologic malignancies: correlation with clinical characteristics. Exp Oncol. 2006;28(3):241-4.

26. Dalamaga M, Karmaniolas K, Panagiotou A, Hsi A, Chamberland J, Dimas $C$, et al. Low circulating adiponectin and resistin, but not leptin, levels are associated with multiple myeloma risk: a case-control study. Cancer Causes Control. 2009:20(2):193-9.

27. Hofmann J, Liao L, Pollak M, Wang Y, Pfeiffer R, Baris D, et al. A prospective study of circulating adipokine levels and risk of multiple myeloma. Cancer Epidemiol Biomark Prev. 2013;22(3):472

28. Esheba NE, Shahba A, El Shora O. Assessment of leptin and resistin levels in non-obese multiple myeloma patients and their relation with Ig level and disease stage. J Egyp Natll Cancer Inst. 2014;26(2):61-6.

29. Hofmann JN, Birmann BM, Teras LR, Pfeiffer RM, Wang Y, Albanes D, et al. Low levels of circulating adiponectin are associated with multiple myeloma risk in overweight and obese individuals. Cancer Res. 2016;76(7):1935-41.

30. Yu W, Cao DD, Li Q, Mei H, Hu Y, Guo T. Adipocytes secreted leptin is a pro-tumor factor for survival of multiple myeloma under chemotherapy. Oncotarget. 2016;7(52):86075-86. 
31. Santo L, Teras LR, Giles GG, Weinstein SJ, Albanes D, Wang Y, et al. Circulating resistin levels and risk of multiple myeloma in three prospective cohorts. Br J Cancer. 2017;117(8):1241-5.

32. Liu Z, Liu H, Li Y, Wang Y, Xing R, Mi F, et al. Adiponectin inhibits the differentiation and maturation of osteoclasts via the mTOR pathway in multiple myeloma. Int J Mol Med. 2020;45(4):1112-20.

33. Salman AS, Salaha ES, Abass MS. Estimation of beta two microglobulins, Fetuin-A, resistin serum level in iraqi multiple myeloma patients. Iraqi J Pharm Sci. 2020:29(2):80-7.

34. Lichtman MA. Obesity and the risk for a hematological malignancy: leukemia, lymphoma, or myeloma. Oncologist. 2010;15(10):1083-101.

35. Harvey $A E$, Lashinger $L M$, Hursting $S D$. The growing challenge of obesity and cancer: an inflammatory issue. Ann N Y Acad Sci. 2011;1229:45-52.

36. Calle EE, Kaaks R. Overweight, obesity and cancer: epidemiological evidence and proposed mechanisms. Nat Rev Cancer. 2004;4(8):579-91.

37. Avgerinos Kl, Spyrou N, Mantzoros CS, Dalamaga M. Obesity and cancer risk: emerging biological mechanisms and perspectives. Metab Clin Exp. 2019;92:121-35

38. Gu L, Cao C, Fu J, Li Q, Li DH, Chen MY. Serum adiponectin in breast cancer: a meta-analysis. Medicine. 2018;97(29):e11433.

39. Pan H, Deng LL, Cui JQ, Shi L, Yang YC, Luo JH, et al. Association between serum leptin levels and breast cancer risk: an updated systematic review and meta-analysis. Medicine. 2018;97(27):e11345.

40. Fang J, Xu X, Mao Q, Ying Y, Zhang X, Xie L. Lower circulating adiponectin is associated with higher risk of renal cell carcinoma: A meta-analysis. Int J Biol Markers. 2020;35(1):57-64.

41. Wang T, Fahrmann JF, Lee H, Li YJ, Tripathi SC, Yue C, et al. JAK/STAT3regulated fatty acid $\beta$-oxidation is critical for breast cancer stem cell self-renewal and chemoresistance. Cell Metab. 2018;27(1):136-150.e135.

42. He JY, Wei XH, Li SJ, Liu Y, Hu HL, Li ZZ, et al. Adipocyte-derived IL-6 and leptin promote breast cancer metastasis via upregulation of lysyl hydroxylase-2 expression. Cell Commun Signal. 2018;16(1):100.

43. Liu Z, Xu J, He J, Liu H, Lin P, Wan X, et al. Mature adipocytes in bone marrow protect myeloma cells against chemotherapy through autophagy activation. Oncotarget. 2015;6(33):34329-41.

44. Parida S, Siddharth S, Sharma D. Adiponectin, obesity, and cancer: clash of the bigwigs in health and disease. Int J Mol Sci. 2019;20(10):2519.

45. Fowler JA, Lwin ST, Drake MT, Edwards JR, Kyle RA, Mundy GR, et al. Host-derived adiponectin is tumor-suppressive and a novel therapeutic target for multiple myeloma and the associated bone disease. Blood. 2011;118(22):5872-82

46. Yang G, Fan W, Luo B, Xu Z, Wang P, Tang S, et al. Circulating resistin levels and risk of colorectal cancer: a meta-analysis. Biomed Res Int. 2016;2016:7367485.

47. Wang CH, Wang PJ, Hsieh YC, Lo S, Lee YC, Chen YC, et al. Resistin facilitates breast cancer progression via TLR4-mediated induction of mesenchymal phenotypes and stemness properties. Oncogene. 2018;37(5):589-600.

48. Pang J, Shi Q, Liu Z, He J, Liu H, Lin P, et al. Resistin induces multidrug resistance in myeloma by inhibiting cell death and upregulating $A B C$ transporter expression. Haematologica. 2017:102(7):1273-80.

49. Lin TC. The role of visfatin in cancer proliferation, angiogenesis, metastasis, drug resistance and clinical prognosis. Cancer Manag Res. 2019;11:3481-91.

50. Zhang H, Zhang N, Liu Y, Su P, Liang Y, Li Y, et al. Epigenetic regulation of NAMPT by NAMPT-AS drives metastatic progression in triple-negative breast cancer. Cancer Res. 2019;79(13):3347-59.

51. Wang YY, Chen HD, Lo S, Chen YK, Huang YC, Hu SC, et al. Visfatin enhances breast cancer progression through CXCL1 induction in tumorassociated macrophages. Cancers (Basel). 2020;12(12):3526.

52. Zhao Q, Li JY, Zhang J, Long YX, Li YJ, Guo XD, et al. Role of visfatin in promoting proliferation and invasion of colorectal cancer cells by downregulating SDF-1/CXCR4-mediated miR-140-3p expression. Eur Rev Med Pharmacol Sci. 2020:24(10):5367-77.

53. Nacarelli T, Fukumoto T, Zundell JA, Fatkhutdinov N, Jean S, Cadungog MG, et al. NAMPT inhibition suppresses cancer stem-like cells associated with therapy-induced senescence in ovarian cancer. Cancer Res. 2020;80(4):890-900

54. Venkateshaiah SU, Khan S, Ling W, Bam R, Li X, van Rhee F, et al. NAMPT/ PBEF1 enzymatic activity is indispensable for myeloma cell growth and osteoclast activity. Exp Hematol. 2013:41(6):547-557.e542.
55. Cagnetta A, Cea M, Calimeri T, Acharya C, Fulciniti M, Tai YT, et al. Intracellular NAD ${ }^{+}$depletion enhances bortezomib-induced anti-myeloma activity. Blood. 2013;122(7):1243-55.

56. Korotchkina L, Kazyulkin D, Komarov PG, Polinsky A, Andrianova EL, Joshi $\mathrm{S}$, et al. OT-82, a novel anticancer drug candidate that targets the strong dependence of hematological malignancies on NAD biosynthesis. Leukemia. 2020;34(7):1828-39.

\section{Publisher's Note}

Springer Nature remains neutral with regard to jurisdictional claims in published maps and institutional affiliations.

Ready to submit your research? Choose BMC and benefit from

- fast, convenient online submission

- thorough peer review by experienced researchers in your field

- rapid publication on acceptance

- support for research data, including large and complex data types

- gold Open Access which fosters wider collaboration and increased citations

- maximum visibility for your research: over $100 \mathrm{M}$ website views per year

At BMC, research is always in progress.

Learn more biomedcentral.com/submissions 